\title{
Introduction to CPM-OFDM: An Energy Efficient Multiple Access Transmission Scheme
}

\author{
Mohammad Irfan, Sang Hoon Lee and Soo Young Shin \\ Wireless Emerging Networking System Lab, Kumoh National Institute of Technology, South-Korea
}

\begin{abstract}
In this paper we propose an energy efficient OFDM transmission scheme. Two CPM (continuous phase modulation) schemes are introduced to OFDM, keeping in view the constant envelope and continuous phase changing nature of CPM modulation. Energy efficiency of the proposed scheme is investigated by simulating PAPR (peak to average power ratio) and is compared with the conventional OFDM scheme. The impact of PAPR on BER is also investigated. BER (bit error rate) of the proposed scheme is simulated in different channels and is compared with conv-OFDM (QPSK-OFDM) scheme.
\end{abstract}

Key words: PAPR, BER, HPA, CPM.

\section{Introduction}

OFDM (orthogonal frequency division multiplexing) is the most popular high data rate and spectral efficient multicarrier communication system. In OFDM the subcarriers are orthogonal to each other, so they will not interfere with each other and more number of subcarriers can be packed to the same spectrum to accommodate multiple users $[1,2]$. It's high data rate, spectral efficiency and robustness against multipath fading make it one of the most promising candidate for portable computing devices and smartphones. These devices demands high data rate but low power consumption as they are usually battery operated.

Despite many advantages of OFDM, high PAPR of an OFDM signal is its major limiting factor in practice. In practical OFDM systems, a HPA (high power amplifier) is used to obtain sufficient transmit power [3]. In a typical OFDM system, the transmit power is only $8 \%$ of the total consumed power, $41 \%$ of power is wasted in HPA and the rest of the circuit consume about $51 \%$ power $[1,4]$.

There are two reasons of $41 \%$ of power loss in HPA,

Corresponding author: Soo Young Shin, professor, research fields: cognitive radio, WLAN, WiMAX. E-mail: wdragon@kumoh.ac.kr. low efficiency of HPA's and high PAPR of OFDM signal which further reduces the efficiency of HPA. HPA has limited linear range, such non-linear effects of HPA's seriously degrades OFDM signal of high PAPR [5]. To solve this problem, the linear range of HPA should be enlarged, which means the IBO (input back off) of HPA should be greater than the PAPR of the signal (unless some pre-distortion technique is used) to avoid such signal distortions $[1,6]$. But increasing IBO means increasing power consumption of HPA and may decrease its efficiency [5]. Improving efficiency of HPA by reducing PAPR of OFDM signal will not only result in power saving, but the probability of bit error will also reduce as the signal will be less distorted.

The $P A P R_{c}$ for a continuous time OFDM transmit signal $x(\mathrm{t})$ is given by Eq. (1), where $N$ represents number of subcarriers, symbol duration is $T, N T$ represents duration of the signal.

$$
P A P R_{c}=10 \log _{10}\left(\frac{\max _{0<t<N T}\left|x(t)^{2}\right|}{1 / N T \int_{0}^{N T}|x(t)|^{2} d t}\right)
$$

Various schemes have been developed to reduce PAPR of OFDM signal. These schemes are summarize in Ref. [7]. Method of coding is discussed in Ref. [8] and tone reservation in Ref. [9]. 
Predistortion scheme in Ref. [10] and clipping in Ref. [11]. All these methods can be divide into two categories distortion and distortionless. Clipping [11], clips the peak power $\max _{0<t<N T}\left|x(t)^{2}\right|$ to some pre-defined value and thus distort the signal which results in high BER at receiver and reduces throughput. The distortion-less methods like tone reservation [9] and selective mapping [12] allows perfect recovery of the original signal at the cost of transmitting some side information which reduces throughput, increase bandwidth requirement and increases signal overhead. Jun and Stuber [13] shown an approach which transforms the OFDM signal into constant envelope by phase modulating the signal after its IDFT (inverse discrete fourier transform). Our approach is different from Ref. [13]. We replaced the conventional modulation schemes used in OFDM such as QPSK, 16-QAM and 64-QAM by GMSK (gaussian minimum shift keying) and MSK (minimum shift keying) (known as scheme 1 and scheme 2 in this work) (Fig. 1).

In our proposed scheme, the input data is first modulated by a CPM modulator, the modulated symbols are then transformed from serial to parallel, an IDFT is then applied to the parallel modulated symbols. After IDFT cyclic prefixes are inserted the data is then again changed from serial to parallel followed by amplification by a HPA and is transmitted, at the receiver side a viterbi detector is used to detect the symbols after parallel to serial conversion.

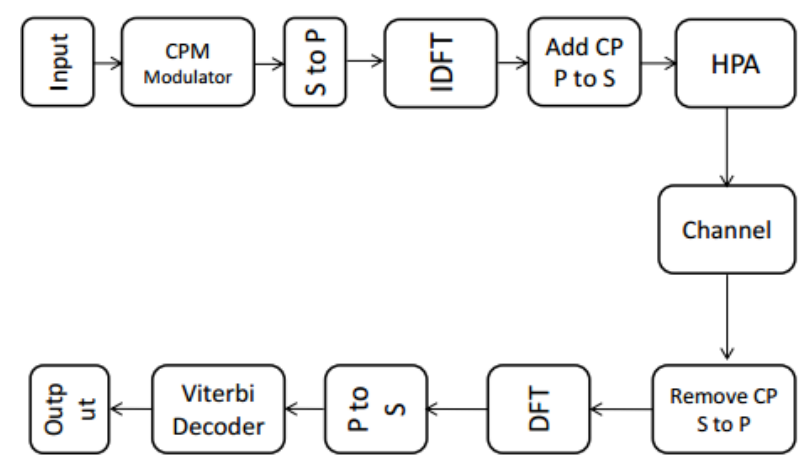

Fig. 1 Proposed CPM-OFDM block diagram.
The rest of the paper is organized as follows: Section 2 presented fundamental of CPM, two CPM modulation schemes that we have selected for this work and some previous work is discussed; Section 3 presented our system model, CPM based OFDM. In Section 3, we designed CPM based transmitter, PAPR analysis of CPM based OFDM, receiver structure for CPM based OFDM and the effect PAPR on BER. In Section 4, we presented our numerical results and conclusion is drawn in Section 5.

\section{Fundamentals of CPM}

As compared to other modulation schemes like QPSK, which is one of the modulation scheme used in LTE [14]. In such modulation schemes, the carrier phase is abruptly reset to zero at the start of every symbol. In QPSK, when the message signal changes by one bit a phase shift of 90 degrees occurs. This discontinuity of QPSK modulated signal lead to high power consumption and some percentage of power occurs outside of the intended band, which leads to poor spectral efficiency [7].

While CPM has constant envelope and the phase of the carrier signal is modulated in a continuous manner, which yields high spectral efficiency [7]. Due to its constant envelop the PAPR of such waveform is unity [7]. A CPM waveform is given by Eq. (2), [15].

$$
S(t ; \beta)=\exp \{\mathrm{j} \phi(\mathrm{t} ; \beta\}
$$

$\phi$ is the phase of the signal and is given by the following equation.

$$
\phi(\mathrm{t} ; \beta)=2 \pi \sum_{i} \beta_{i} h_{i} q(t-i T)
$$

$\beta=\beta_{i}$ represents the discrete time symbol sequence of $M$-ary symbols form $i_{\text {th }}$ user each symbol carries $m=\log _{2} M$ bits, $T$ is the symbol duration, $h_{i}$ is the modulation index. Smaller the modulation index the more narrower bandwidth the signal occupies. $M$ is the value of M-ary signaling. Throughout this work is $M=4$. Two CPM schemes are selected for this work.

Scheme 1: 4-ary signaling is used, modulation 
index $h_{i}=0.3$, Raised cosine frequency pulse with Pulse length $\mathrm{L}=4$ and minimum square Euclidean distance, $d_{\text {min }}{ }^{2}=1.48$.

Scheme 2: 4-ary signaling with modulation index $h_{i}$ $=0.6$, Gaussian frequency Pulse with $\mathrm{BT}=0.25$, Pulse length, $\mathrm{L}=3$, and minimum squared Euclidean distance, $d_{\text {min }}^{2}=4.6$.

The above two scheme differs in PAPR and BER performance. Scheme 1 has smaller modulation index than scheme 2, so it will occupy a narrow bandwidth as compare to scheme 2, its power spectral density will drop more abruptly, which will result in better PAPR performance. But on the other hand, scheme 1 has small value of $d_{\min }^{2}$, so Scheme 2 will have better BER performance compare to Scheme 1. Increasing $d_{\text {min }}^{2}$, lowers the probability of bit error [15].

\section{CPM Based OFDM}

Consider an OFDM system as shown in Fig 1. $N$ number of symbols and sub-carriers. Each symbol is mapped to one sub-carrier. In our proposed scheme, the input data is first modulated by a CPM modulator. The modulated samples are then converted from serial to parallel by a serial to parallel converter as shown in Fig 1.

$$
\mathrm{S}_{i}=\left[S_{\{\mathrm{i}, 0\}}, S_{\{\mathrm{i}, 1\}} \ldots \ldots \ldots \ldots, S_{\{\mathrm{i}, \mathrm{N}-1\}}\right]^{T}
$$

where, $i$ is the index of a user.

$$
S_{i, l}=\exp \{\mathrm{j} \phi(\mathrm{i} ; 1)\}
$$

This is discrete time equivalent of Eq. (2). The continuous time transmitted OFDM signal $x(t)$ is expressed as:

$$
\mathrm{x}(\mathrm{t})=\frac{1}{\sqrt{N}} \sum_{k=0}^{N-1} \mathrm{X}_{k} \mathrm{e}^{j 2 \pi k \Delta f t}, 0 \leq t \leq T
$$

where, $T$ is the duration of the OFDM symbol, $X_{k}=$ $\left[X_{0}, X_{1}, \ldots X_{N-1}\right]$ is the input data block, $\Delta f=1 / \mathrm{T}$ is the frequency spacing between two adjacent subcarriers. The same way a discrete time CPM-OFDM signal $x(n)$ with Nyquist rate is obtained by $N$-point IDFT operation of $S_{\{(i, l)\}}$.

$$
\mathrm{x}(\mathrm{n})=\frac{1}{\sqrt{N}} \sum_{k=0}^{N-1} \mathrm{~S}_{(i, l)} \mathrm{e}^{j 2 \pi \frac{k n}{N}}, \mathrm{n}=0,1 \ldots N-1
$$

The equation above shows the waveform of a CPM based OFDM. After insertion of cyclic prefix and digital to analog conversion the signal is amplified by a HPA (high power amplifier) and is transmitted.

\subsection{PAPR Analysis}

PAPR stands for Peak to Average Power Ratio. It is basically used as performance metric for envelop variations in a signal. As OFDM is a multi carrier system, a set of orthogonal frequencies are used.

Mathematically these frequencies will not interfere with each other, but in practice they interfere with each other. In this paper, $N$ number of sub carriers are used. Assuming amplitude of each sub-carrier is 1 , as the constellation points are taken from MSK and GMSK modulators. These sub carriers can constructively add up resulting in high peak powers. So the range of PAPR is 1 to $N$. In case of non unity amplitude A like the case of QAM, the max PAPR is given by equation below [16].

$$
P A P R_{\text {max }}=A_{\text {max }}^{2} N
$$

Another reason of high PAPR of an OFDM signal is the spectral leakage of DFT. The sampling period $\Delta t$ $=1 /$ fs. The $n$th array element in frequency domain is known as bin. The frequency of bucket $\mathrm{n}$ can be related to the frequency of input signal $\mathrm{S}\{i, 1\}$ by equation below [17].

$$
f_{n}=\frac{n}{N \Delta t}
$$

where, $n$ is the number of bin, $N$ is the number of samples and $f_{n}$ is the frequency of bin $n$. Rearranging

$$
n=\frac{N f_{n}}{f_{s}}
$$

$N$ is the total number of sub-carriers which is equal to 16 for this work, $f_{s}$ sampling frequency and $f_{n}$ is the frequency of $n$th bin. For $900 \mathrm{~Hz}$ signal the maximum amplitude occurs at $n=N f_{n} / f_{s}=16^{*} 900 / 3000=4.8$. As each bin represents a frequency not a range of frequencies and bin number is an integer, then the 
question is how can DFT represent energy at bin number 4.8 , actually it shows some leakage of energy between bin 4 and bin 5 . In DFT for some frequencies there is some energy leakage between bins, while in other frequencies, there is no energy leakage. For example, for a $1,500 \mathrm{~Hz}$ waveform, $n=N f_{n} / f_{s}=$ $16^{*} 1500 / 3000=8$, there is no spectral leakage between bin 8 and bin 7 or bin 8 and bin 9. In OFDM multi-carriers are generated by IDFT and due to the spectral leakage problem of DFT and interference of the multi carriers OFDM exibits high PAPR. As the input $S\{i, 1\}$ is random so PAPR of $x(\mathrm{n})$ given by equation below is also a random variable.

$$
P A P R=10 \log _{10}\left(\frac{\max _{0<t<\tau^{*} N-1}\left|x(\mathrm{n})^{2}\right|}{E\left[x(n)^{2}\right]}\right)
$$

Therefore CDF (cumulative distribution function) is used to describe its properties.

$$
\begin{aligned}
& C D F=\operatorname{Prob}(P A P R \leq \gamma) \\
& \operatorname{Pr} o b(P A P R \leq \gamma) \approx 1-\left(1-e^{\gamma}\right)^{N}
\end{aligned}
$$

where, $\gamma$ is a constant. PAPR of a discrete time signal $x(\mathrm{n})$ is an accurate approximation of the continuous time PAPRc, if the up-sampling factor $\tau \geq 4$, [7]. For this work we are using up-sampling factor $\tau=4$.

\subsection{Effect of PAPR on BER Performance}

In this section, we determine the relation of PAPR and BER performance of an OFDM signal in the absence of IBO from HPA. A HPA exibits two kinds of distortions, AM-AM and AM-PM distortion. When the HPA is operating in the linear region, the output voltage is only a scaled version of the input voltage. However when the input signal has high peaks in the compression region, the output of the HPA is amplitude distorted. The resultant distortions are known as AM-AM distortions. Similarly AM-PM is used for phase non linearity. In such kind of distortion the phase of the signal is distorted. But here we will only consider the effect of AM-AM distortion. The efficiency $\eta$ of HPA depends on the input voltage or input power. An amplifier operates more efficiently if the input is within the range of operating point. CPM has constant envelop, but due to the PAPR problem of multi-carrier systems there is a large envelope fluctuation. So the efficiency $\eta$ depends on PAPR and class of the amplifier [16].

$$
\eta=G \times e^{-g \times P A P R_{d B}}
$$

where, $G$ is the gain of an amplifier. As the probability of bit error depends on SNR of the signal.

$$
S N R=\frac{\eta \times[x(n)]^{2}}{n_{o}{ }^{2}} \times T
$$

where, $n_{\mathrm{o}}{ }^{2}$ represents noise power and $[x(\mathrm{n})]^{2}$ is the transmitted signal power SNR depends on the efficiency $\eta$ of HPA. And the efficiency of HPA depends on PAPR of the signal. So the probability of bit error depends on PAPR of the signal [16].

$$
\begin{gathered}
\text { SNR }=\frac{G}{\xi} \times \frac{x(n)^{2} \times T}{n_{o}{ }^{2}} \\
\xi={ }_{P A P R}(10 \mathrm{~g} / \ln 10) \\
P A P R \leq \text { SatLevel } \\
P A P R>\text { SatLevel }
\end{gathered}
$$

Eq. (15) shows that low PAPR results in better HPA efficiency and high SNR which results in low energy consumption by HPA and low error rates.

\section{Numerical Results}

\subsection{PAPR Numerical Results}

In this section, we first numerically analyze PAPR and BER properties of our proposed two schemes of CPM-OFDM and then compare them to conventional OFDM scheme. Relation of PAPR to BER in the absence of IBO is also numerically analyzed in this section. All the simulations performed in this section consists of $(N=16) N=$ number of allocated sub carrier to one user. Number of users $=1$; Fig. 2 shows numerically calculated CDF of PAPR of our two proposed schemes and conventional OFDM. Due to low modulation index of scheme 1 its power spectral density will drop abruptly as compare to scheme 2 of high modulation index and conventional OFDM. 
Therefore, scheme 1 has low PAPR as compare to scheme 2 and conventional OFDM. The previous discussion in section 2, PAPR analysis shows that CPM-OFDM will have constant envelop but as Fig. 2 shows CPM-OFDM still has envelope variations. The reasons are already discussed in the Section 2. Spectral leakage and sub-carriers interference which results in high peaks. PAPR statistics of these three schemes are provided in Table 1. Proposed scheme 1 has almost $4 \mathrm{~dB}$ performance gain over conv-OFDM and almost $0.5 \mathrm{~dB}$ performance gain over scheme 2 in terms of their max PAPR. In order to operate the HPA in linear region, so that it will not distort the OFDM signal, the input back off must be higher than the PAPR of the signal. Input back-off is the amount of power drawn from dc (direct current) source of HPA, in order to keep the HPA in linear region. Table 2 shows required amount of minimum input back-off calculated from CDF plot of Fig. 2. Table 2 shows

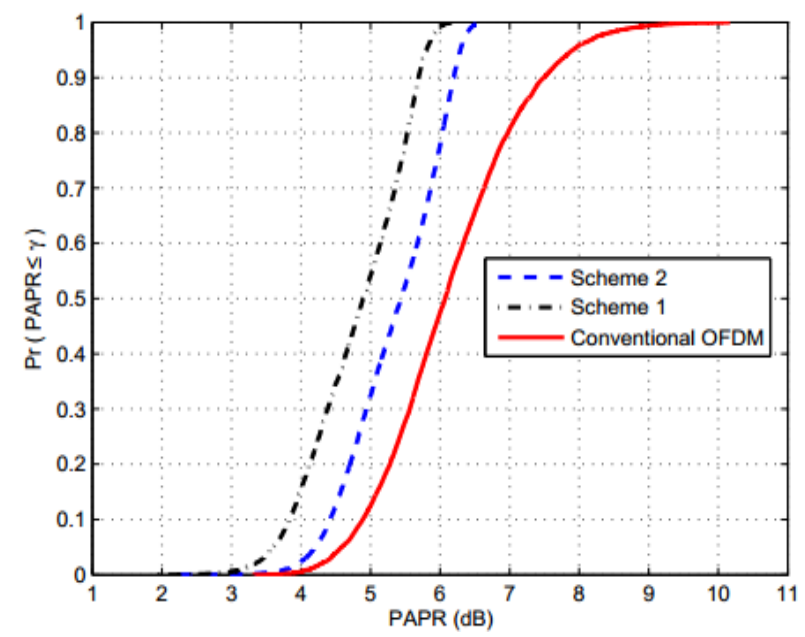

Fig. 2 PAPR plots of scheme 1,2 and conv-OFDM.

Table 1 PAPR statistics of Fig. 2.

\begin{tabular}{llll}
\hline Scheme & Max [dB] & Min [dB] & Mean [dB] \\
\hline Scheme 1 & 6.23 & 1.95 & 4.82 \\
Scheme 2 & 6.68 & 2.24 & 5.36 \\
Conv-OFDM & 10.16 & 3.32 & 6.14 \\
\hline
\end{tabular}

Table 2 Required IBO from CDF plot.

\begin{tabular}{lll}
\hline Scheme & IB-90\% $[\mathrm{dB}]$ & IB-99\%[dB] \\
\hline Scheme 1 & 5.5 & 6 \\
Scheme 2 & 6.3 & 6.5 \\
Conv-OFDM & 7.6 & 9.4 \\
\hline
\end{tabular}

that scheme 1 will draw little amount of power from the dc source which happens to be the most power efficient scheme of all the three schemes.

\subsection{PAPR effect on BER}

Numerical results of PAPR and BER relation are presented in this section. Analytically it is already developed in the previous section (18). Three different HPA with input saturation level of 2, 4 and $6 \mathrm{~dB}$ are considered here. The output of amplifier is saturated and distorted, once the input cross it saturation limit. for such kind of HPA with no IBO, BER is simulated and is shown in Fig. 3. The Fig. 3 shows that BER is at low when the input signal PAPR is with in HPA input saturation level. As the input signal PAPR crosses the input saturation of HPA the probability of bit error increases abruptly. The probability of bit error increases exponentially with increasing PAPR.

\subsection{Bit Error Rate (BER) Performance}

Fig. 4 shows BER performance of Scheme 1, Scheme 2 and conv-OFDM in AWGN channel. The effect of HPA distortions to OFDM signal are also added in the form of IB-90\% from Table 2. The actual BER performances are plotted by solid lines for different channels. The BER performance with HPA distortions are plotted by dotted lines as a function of $\mathrm{E}_{\mathrm{b}} / \mathrm{N}_{\mathrm{o}}+\mathrm{IB}_{90 \%}$. As shown without taking the effect of HPA non-linearity, scheme 2 has better BER performance

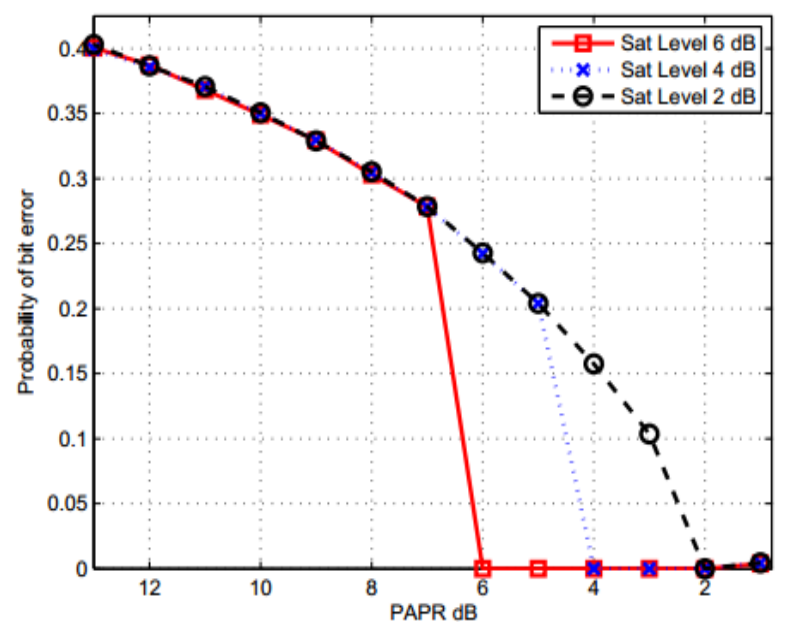

Fig. 3 Effect of PAPR on BER, under SNR $=10 \mathrm{~dB}$. 


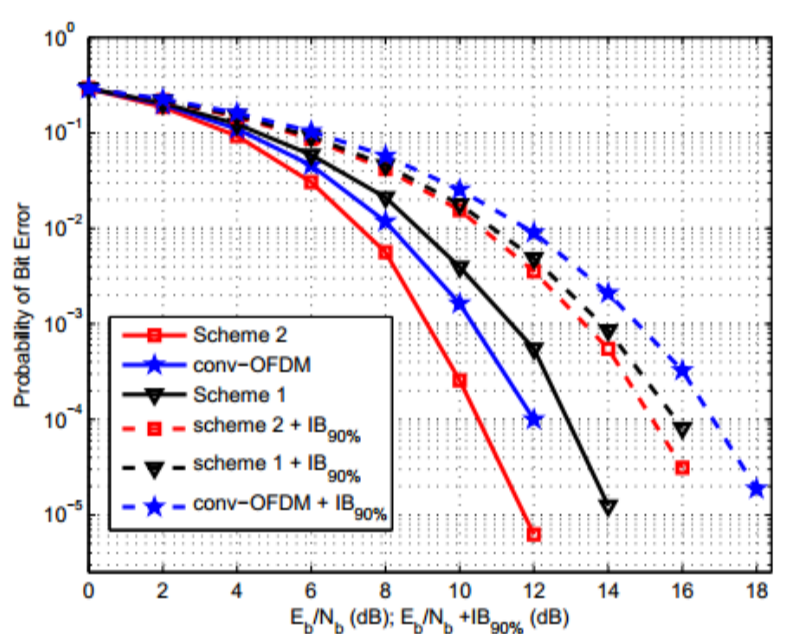

Fig 4 BER Plots of scheme 1, 2 and Conv-OFDM in AWGN Channel.

as compare to scheme 1 and Conv-OFDM. The reason is the large minimum square Euclidean distance $\mathrm{d}_{\min }{ }^{2}=$ 4.6, of scheme 2 as compared to $\mathrm{d}_{\min }{ }^{2}=1.48$ of scheme 1. At BER of 10-4 in AWGN channel scheme 2 has almost $1.5 \mathrm{~dB}$ gain over conv-OFDM and almost $2 \mathrm{~dB}$ gain over scheme 1 . When the HPA non-linearity is taken into account in the form of $\mathrm{E}_{\mathrm{b}} / \mathrm{N}_{\mathrm{o}}+\mathrm{IB}_{90 \%}$,we notice that scheme 1 and scheme 2 have almost the same BER performance, although the previous discussion shows that scheme 2 has better BER performance. Scheme 2 has better BER performance than scheme 1, but it has high PAPR as well, so in presence of HPA non-linearity, both the scheme has almost the same BER performance. The conv-OFDM shows the worse performance of all due to its high PAPR as compare to scheme 1 and scheme 2 .

\section{Conclusion}

In this work, we have compared PAPR properties of our proposed two schemes with conv-OFDM, and have shown that how the proposed schemes will have better PAPR performance as compare to conventional scheme. High PAPR results in worse BER performance in the absence of HPA Input Back-off, and high power consumption and low efficiency in the presence of input Back-off. BER performance is also evaluated in the presence and absence of HPA non-linearity. In the presence of HPA non-linearity which is more realistic approach our proposed scheme outperforms the conv-OFDM (QPSK-OFDM).

\section{Acknowledgment}

This research was supported by the MSIP(Ministry of Science, ICT and Future Planning), Korea, under the ITRC(Information Technology Research Center)) / CITRC(Convergence Information Technology Research Center) support program (IITP-2015-H8601-15-1011) supervised by the IITP(Institute for Information \& communications Technology Promotion).

\section{References}

[1] Abraham, P., and Ruiz, A. 1980. "Frequency Domain Data Transmission Using Reduced Computational Complexity Algorithms." Presented at the IEEE International Conference on Acoustics, Speech, and Signal Processing.

[2] Saltzberg, B. 1967. "Performance of an Efficient Parallel Data Transmission System.” IEEE Transactions on Communication Technology 15: 805-11.

[3] Tao, J., Li, C., and Ni, C. X. 2013. "Effect of PAPR Reduction on Spectrum and Energy Efficiencies in OFDM Systems with Class-A HPA over AWGN Channel." IEEE Transactions on Broadcasting 59: 513-19.

[4] Tao, J. 2007. "Multicast Broadcast Services Support in OFDMA-Based WiMAX Systems [Advances in Mobile Multimedia]." IEEE Communications Magazine 45: 78-86.

[5] Krongold, B. S., and Douglas, L. J. 2004. "An Active-Set Approach for OFDM PAR Reduction via Tone Reservation." IEEE Transactions on Signal Processing 52: 495-509.

[6] Green, W., Marilynn, P., Perrins, E., and Svensson, T. 2011. "Introduction to CPM-SC-FDMA: A Novel Multiple-Access Power-Efficient Transmission Scheme." IEEE Transactions on Communications 59: 1904-15.

[7] Hee, H. S., and Lee, J. H. 2005. "An Overview of Peak-to-Average Power Ratio Reduction Techniques for Multicarrier Transmission." IEEE Wireless Communications 12: 56-65.

[8] Davis, J. A., and Jonathan, J. 1999. "Peak-to-Mean Power Control in OFDM, Golay Complementary Sequences, and Reed-Muller Codes." IEEE Transactions on Information Theory 45: 2397-417.

[9] Krongold, B. S., and Douglas, L. J. 2004. “An Active-Set Approach for OFDM PAR Reduction via Tone 
Reservation." IEEE Transactions on Signal Processing 52: 495-509.

[10] Andrea, D., Aldo, N., Vincenzo, L., and Ruggero, R. 2001. "Nonlinear Predistortion of OFDM Signals over Frequency-Selective Fading Channels." IEEE Transactions on Communications 49: 837-43.

[11] Armstrong, J. 2002. "Peak-to-Average Power Reduction for OFDM by Repeated Clipping and Frequency Domain Filtering." Electronics letters 38: 246-47.

[12] Lee, Y. L. 2003. "Peak-to-Average Power Ratio in MIMO-OFDM Systems Using Selective Mapping." IEEE Communications Letters 7: 575-77.

[13] Jun, T., and Stuber, G. L. 2005. "Frequency-Domain
Equalization for Continuous Phase Modulation." IEEE Transactions on Wireless Communications 4: 2479-90.

[14] Chen, Y. 2009. "Research on the Modulation and Coding Scheme in LTE TDD Wireless Network." Presented at the International Conference on Industrial Mechatronics and Automation.

[15] Anderson, J. B., Aulin, T., and Sundberg, C. E. 1986. Digital phase modulation. Springer.

[16] Dov, W. L. 2005. "Definition of Efficient PAPR in OFDM.” IEEE Communications Letters 9: 832-34.

[17] Lyon, D. A. 2009. "The Discrete Fourier Transform, Part 4: Spectral Leakage.” Journal of object technology 8. 